\title{
Políticas públicas y gobernanza: Articulación para una gestión pública local autónoma
}

\author{
Hilarión Vegas Meléndez \\ Universidad Católica del Ecuador, Manabí, Ecuador \\ Email: hvegasm@gmail.com
}

\begin{abstract}
Resumen: Este trabajo busca referenciar espacios de acercamiento entre las políticas públicas y la gobernanza, revelando instrumentos metodológicos que permiten mostrar el alcance de la entrevista en profundidad y el método delphi como estrategias de recolección de información para la posterior construcción de una teoría sustantiva; los informantes están conformados por alcaldes (actores sociales) e investigadores (expertos) cuyo aporte representa una significativa experiencia para las ciencias sociales en cuanto a la estructuración de una ruta metodológica entre la interpretación del discurso -hermenéutica investigativa- y lo empírico, emanando códigos "in vivo” que posteriormente permitieron la construcción de categorías que contribuyeron establecer un recorrido que permite articula políticas públicas construidas desde la base de la sociedad en corresponsabilidad con la gestión gubernamental. Las principales conclusiones y hallazgos permiten identificar lo cotidiano como factor emergente de políticas públicas locales, así como la importancia de la participación de influyentes en la confección del ecosistema local.
\end{abstract} pación

Palabras claves: Política pública, gobernanza, gestión pública, partici-

\section{Public policies and governance: Articulation for autonomous local public management}

\begin{abstract}
This paper seeks to index spaces between public policy and governance, revealing methodological tools that allow the presentation of the scope of the in-depth interview and the delphi method as information collection strategies for the subsequent construction of a substantive theory; the informants are made up of mayors (social actors) and researchers (experts) whose contribution represents a significant experience for the social sciences in terms of structuring a methodological path between the interpretation of the investigative-hermeneutical discourse and the empirical one, coming from "in live" code that later allowed the construction of categories that contributed to establish a route that allows articulating public policies built from the base of society in co-responsibility with government management. The main conclusions and findings allow us to identify routine as an emerging factor of local public policies, as well as the importance of the participation of influential people in the confection of the local ecosystem.
\end{abstract}

Keywords: Public policy, governance, public management, participation 


\section{Políticas públicas e governança: Articulação para administração pública local autônoma}

Resumo: Este trabalho busca referenciar espaços de abordagem entre políticas públicas e governança, revelando instrumentos metodológicos que permitem mostrar o escopo da entrevista em profundidade e o método de Delphi como estratégias de coleta de informações para a subsequente construção de uma teoria substantiva; os informantes são constituídos por prefeitos (atores sociais) e pesquisadores (especialistas) cujo contributo representa uma experiência significativa para as ciências sociais em termos de estruturação de uma via metodológica entre a interpretação do discurso - a hermenêutica investigativa e os códigos empíricos e emanantes “ in vivo "que mais tarde permitiu a construção de categorias que contribuíram para estabelecer um caminho que permita articular políticas públicas construídas a partir da base da sociedade em corresponsabilidade com a gestão do governo. As principais conclusões e descobertas permitem identificar o quotidiano como um fator emergente das políticas públicas locais, bem como a importância da participação de pessoas influentes na edificação do ecossistema local.

Palavras-chave: política pública, governança, gestão pública, participação

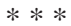

\section{Introducción}

Para muchos el proponer políticas públicas basadas en la realidad local significaría algo natural cuando la descentralización político-administrativa funciona sin ningún tipo de inconvenientes en sociedades responsables de su destino y de alto compromiso en la gestión de lo público; sin embargo, para otros, esta algo lejos el implementar políticas públicas que surjan de las bases del diálogo representativo producto del acercamiento ente las instituciones de poder y la ciudadanía organizada, tal como es el caso de los gobiernos locales en Venezuela, donde se imposibilita el diseño de políticas públicas focalizadas u orientadas al desarrollo de lo local, esto debido a la ausencia de leyes y normas que le permitan hacer ejercicios de gobernanza mas autónomo y sin ningún tipo de contratiempos; según el Banco Mundial en su informe de 1993, la gobernanza es "la manera en que se ejerce el poder en el manejo de los recursos económicos y sociales para el desarrollo, refiriéndose al poder de los gobiernos para definir y resolver las políticas relacionadas con el desarrollo” (Banco Mundial, Informe 1993, p. 2), aquí se observa la importancia que tiene el poder gubernamental para incluir y decidir sobre la posibilidad de diseñar políticas públicas de bienestar y desarrollo.

Por otro lado, la gobernanza (Cátala Prats, 2001, p, 21) “puede verse como la pauta o estructura que emerge en un sistema sociopolítico como el resultado conjunto de los esfuerzos de interacción de todos los actores intervinientes”, esta definición puede ser confundida con la gobernabilidad, aunque ambas se interrelacionan, se les debe saber separar en cuanto a su origen y finalidad; donde la gobernanza se caracteriza por ser producto de 
los acuerdos entre la sociedad y el poder, utilizando métodos que garanticen reglas donde todos ganan.

La estructura de gobernanza político-administrativa venezolana esta propuesta en la Constitución de la República Bolivariana de Venezuela2000 a lo largo de todo su contenido, en la que se asume al Consejo Federal de Gobierno, el Consejo Estadal de Coordinación y Planificación de Políticas Públicas, y el Consejo Local de Planificación de Políticas Públicas, en ellas están representadas las tres vertientes del poder público en Venezuela: República (Nacional), Estadal (Regional) y Municipal (Local); y aun cuando todas se mantienen vigentes, su proceder se ha visto muy minimizado por la aplicación de leyes y normas impulsadas desde el ejecutivo nacional en las que buena parte de sus competencias se han diluido en la burocracia, revocado por la re-centralización y caído en la inercia producto la ausencia de una legislación que garantice la autonomía de sus decisiones; sin dudas, afectando con mayor grado a las localidades dado su alto grado de dependencia por los recursos que requiere les sean transferidos, y que en muchas ocasiones reclaman gestos samaritanos del gobierno central para poder gestionar sus responsabilidades y deberes a tiempo en favor de sus comunidades, y por supuesto debilitando su capacidad de respuesta como gestión pública local.

Por su parte, la gestión pública es una estructura procedimental de acción gubernamental que lleva tareas de servicio a la sociedad a través de mecanismos legales y de ejecución política denominadas en muchas ocasiones como políticas públicas, cuya orientación final es la de servir a la ciudadanía garantizándole calidad de vida. Para ello, a lo largo de diversas etapas de revisión y reestructuración, la gestión pública ha focalizado sus acciones en la procura de espacios de acompañamiento por parte del ciudadano en la construcción de una agenda pública que permita conocer sus necesidades y la raíz de los problemas focales que permean las posibilidades de desarrollo social. En este sentido, se busca constantemente fortalecer las localidades con el objeto de llegar con más exactitud al conocimiento de los problemas sociales desde la óptica comunitaria; el espacio local es el lugar más cercano del ciudadano al poder constituido, y parte de su importancia radica en la contribución directa de la gente a la solución de sus problemas en virtud del conocimiento del mismo y con la probabilidad de conocer potencialmente cual es la mejor solución a ejecutarse.

Este trabajo muestra aspectos breves, a través de una serie de reflexiones y hallazgos encontradas en la investigación de quien suscribe denominada Gestión pública local, gobernanza y participación: Una visión a partir de los actores sociales en Venezuela, sobre la problemática existente en diversas localidades del contexto venezolano en cuanto a las debilidades de sus políticas públicas para brindar soluciones a problemas no focalizados, la poca efectividad de la gobernanza local en auspiciar soluciones mancomunadas o consensuadas, así como del estreñimiento de la gestión pública local en brindar calidad de vida a los ciudadanos de manera proyectada y con visión competitiva. 


\section{El sendero investigativo}

La investigación está enmarcada en una perspectiva interpretativa y de carácter cualitativo. Ello significa, que se asume el paradigma inductivointerpretativo en una concepción hermenéutica para abordar el sujeto de estudio. Esto permite, dada su flexibilidad, un proceso interactivo continúo entre el investigador y el objeto de estudio en el levantamiento de la información. El referente epistémico es la teoría fundamentada, la cual ofrece una serie de estrategias de análisis de información empírica, cuyo procesamiento a través de sus distintas fases admite la posibilidad de construcción de una teoría.

Es significativo entender que este estudio dentro del ámbito de lo público, especialmente desde la perspectiva de la gestión local, permite comprender el fenómeno como se desarrolla cotidianamente; lo que conlleva a que la búsqueda del conocimiento se dé desde un esquema inductivo, generando la teoría a partir del discurso de los actores sociales, partiendo desde "lo particular a lo general en pequeños pasos, más bien que en grandes movimientos" (Diesing, 1971, p. 233). Este enfoque cualitativo representa una ventaja dada su flexibilización; lo que conlleva que "la investigación cualitativa produce hallazgos a los que no se llega por medio de procedimientos estadísticos u otros medios de cuantificación" (Strauss y Corbin, 2002, pp. 11-12); así como "es más probable que aparezcan nuevos conceptos cuando se experimentan nuevos fenómenos” (Eisner, 1998, p. 42).

La investigación focaliza su perspectiva siguiendo la fuente microsociológica, centrada en la vida cotidiana, tal como se considera desde el enfoque dramatúrgico al "comprender la acción social a la luz de la metáfora teatral (actores sociales, papeles, escenario, backstage...)” (Goffman, 1959, p. 32). Esta experiencia permite advertir las experiencias de los principales actores en la gestión pública local mediante la observación en los eventos de transformación social en la que se generaron, así como sus actuales derivaciones; es importante considerar que esta investigación implica la captación -mediante la interpretación y el diálogo- del sentido de lo que el otro o los otros quieren decir con sus palabras o silencios, con sus acciones o inmovilidades; es decir, el uso continuado de la hermenéutica como instrumento complementario para la reflexión generadora de comprensión e interpretación del discurso generado en la interacción micro-sociológica con los actores sociales seleccionados, como es el caso de Alcaldes de diferentes localidades; en este aspecto es importante acotar que la hermenéutica aplicada a las investigaciones en el área de las ciencias sociales facilitan una comprensión de los fenómenos socio-políticos, permitiendo su interpretación de una manera mas objetiva.

Para ello, fue importante revisar la postura de que "sí todo discurso se actualiza como acontecimiento, todo discurso es comprendido como sentido" (Ricoeur, 1969, p. 26); donde la experiencia está representada en la expresión, asumiendo el intercambio intersubjetivo en sí, y la comunicación con el receptor. Lo que se comunica en el acontecimiento del habla no es la 
experiencia del hablante como ésta fue experimentada, sino su sentido. La experiencia vivida permanece en forma privada, pero su significación, su sentido, se hace público a través del discurso (Ibíd., p. 27). A su vez, es necesario resaltar que "el lenguaje no es sólo una de las dotaciones de que está pertrechado el hombre tal como está en el mundo, sino que en él se basa y se representa el que los hombres simplemente tengan mundo..." (Gadamer, 1988, p. 56), lo cual permite reforzar la experiencia de intercambio con los actores sociales en interacción micro-sociológica permitiendo que los mismos expresen sus experiencias y conocimientos sobre la gestión pública, mostrando su peculiar forma de trascendencia en el ámbito de lo local.

Por otro lado, mediante la perspectiva cualitativa se logró encontrar y analizar un área problemática -con su debida delimitación- por medio de una indagación y revisión de naturaleza teórica realizada en profundidad y de manera sistematizada a través del uso de fuentes escritas. Así como también, un acercamiento con investigadores -utilizando el Método Delphi ${ }^{1}$ y entrevistas en profundidad a actores de la gestión pública local, tal como es el caso de alcaldes en ejercicio, cuya experiencia representa una valiosa contribución al presente estudio, así como también lo puede hacer cualquier otro actor social que pueda brindar una apreciable información.

En la investigación fueron tomadas en consideración cuatro municipios, representados por sus respectivos gobiernos locales: dos (2) de la región centro occidental (Mérida; Municipio Libertador y Táchira; Municipios Lobatera / Michelena), estados andinos cuyas costumbres sociales y tradiciones agrícolas permanecen intactas como modo de vida local; uno (1) de la región Capital (Miranda; Municipio Independencia), cuya fortaleza se centra en la geografía y su cercanía con la Capital de la República (Caracas), representando un emblemático municipio en el corazón de los Valles del Tuy. Luego de las entrevistas en profundidad a los actores sociales, se dio a lugar una serie de hallazgos, procediéndose a "extraer" los llamados “códigos in vivo" (ver Cuadro 1) identificados como aportes que el actor social brinda a través de la conversación, y que el investigador considera de suma importancia para la construcción de las categorías, generándose incluso sub-categorías que ayudan a dimensionar cada una de ellas, para posteriormente agrupar en familias; con ello se facilita el tránsito entre la teoría sustantiva a la teoría formal.

Es de hacer notar que este proceso de construcción de la teoría se incorporó la postura de los expertos (visión de los scouts), cuyo aporte revela aspectos novedosos a la investigación. En la triangulación de la información y del resultado interpretativo, al proceder denominar (categorización), el investigador procuro establecer una teoría ajustada a los hallazgos encontrados, aplicando el uso de estrategias propuestas por la teoría fundamentada la cual "es una metodología de análisis, unida a la recogida de datos, que utiliza un conjunto de métodos, sistemáticamente aplicados, para generar una teoría inductiva sobre un área sustantiva” (Glaser, 1978, p.30). 
Es necesario destacar que existen ciertas similitudes en los que la teoría fundamentada se acerca con otras tendencias de investigación cualitativa. Sin embargo, se diferencia de la mayoría de ellas, por su énfasis en la construcción y el desarrollo de un tipo de teoría que se denomina sustantiva, donde la misma (De La Torre, 2004, p.5) "se edificaría en torno a ámbitos muy delimitados o particulares de la realidad social o cultural sobre los cuales, la teoría formal no ha desarrollado una comprensión y una conceptualización específica“.

La codificación de los datos recogidos, posteriormente fueron analizados para llegar a una teoría; siendo en este caso la etiqueta (lenguaje en vivo) parte de la codificación, así como también el “código en vivo” (símbolos claves expresados por los actores), para luego de allí trasladarse a la codificación axial, cuya funcionalidad se da para relacionar varias categorías en torno a una sola categoría que sirve de eje, pasando de allí a una matriz condicional-secuencia, tal como se muestra en el cuadro 1:

\section{Cuadro 1. \\ Codificación de los datos recogidos}

\begin{tabular}{|c|c|c|c|c|c|}
\hline Citas textuales & $\begin{array}{l}\text { Códigos } \\
\text { extraidos }\end{array}$ & Categorias & $\begin{array}{c}\text { Sub- } \\
\text { Cattegorias }\end{array}$ & Familias & Visión del Scout \\
\hline 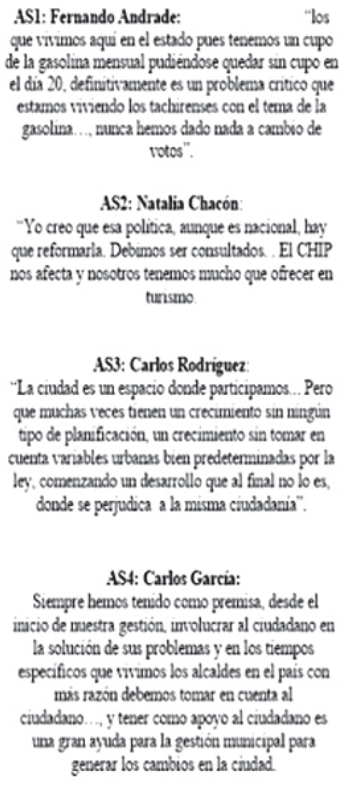 & $\begin{array}{l}\text { Acciones de gobierno } \\
\text { que intolucten } \\
\text { construcción de } \\
\text { agenda publica para lo } \\
\text { local } \\
\text { Identificacion. } \\
\text { interpretaciony } \\
\text { pronizacion de } \\
\text { pecesidades crecieates } \\
\text { junto al cuudadano }\end{array}$ & 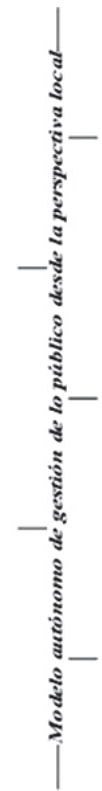 & $\begin{array}{c}\text { Vision } \\
\text { ciudadana } \\
\text {. Vision } \\
\text { institucional }\end{array}$ & 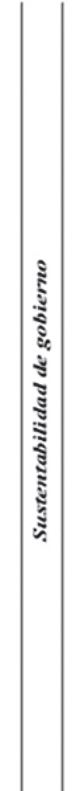 & 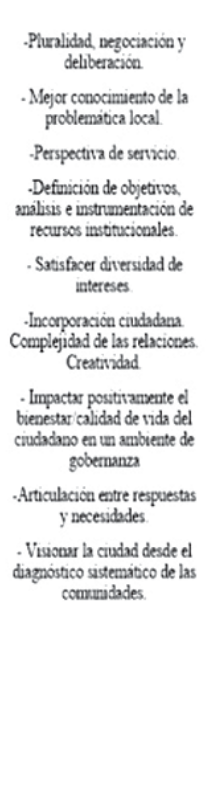 \\
\hline
\end{tabular}

Fuente: Elaboración propia (2015) 
Este cuadro 1 muestra una de las categorías resultantes: Modelo autónomo de gestión de lo público desde la perspectiva local, producto del proceso de "extracción", allí se observa el proceso de saturación y vinculación teórica de los entrevistados con los investigadores expertos consultados (Método Delphi), en las que se "cruzan datos" (triangulación) y quedando a juicio del investigador la construcción de la categoría, producto de la asociación entre las familias resultantes, siendo en este caso sustentabilidad de gobierno la cual que se asocia con los hallazgos incorporados; así como la interpretación dada por quien valora estos resultados.

\section{Acercamientos desde la hermenéutica investigativa}

Desde el espacio investigativo es importante destacar la aplicación del método Delphi a connotados investigadores quienes presentan sus reflexiones e interpretaciones sobre los temas relacionados con las dimensiones expuestas (políticas públicas, gobernanza y gestión pública local), cuyo aporte es representativo y vinculante a los objetivos de este trabajo. Cabe destacar que las dimensiones política pública y gobernanza fueron analizadas por los investigadores, dejando la dimensión gestión pública local a los alcaldes (actores sociales) a través de la entrevista en profundidad.

\section{Política pública}

Una política pública "representa un conjunto de acciones/actividades enmarcadas en normas y procedimientos regulatorios cuya orientación está dada a satisfacer necesidades colectivas, promovidas por el Estado y por la presión social” (Vegas, 2009, p. 31), esta definición deja tres elementos relevantes: 1.- Normas y procedimientos regulatorios, esto conlleva a la legalidad de la acción/actividad; ahora bien, hasta donde es legítima si no es validada por quienes reciben el beneficio 2.- Necesidades colectivas, es una invitación a conocer las raíces del problema, identificándolas desde la participación de quien las padece, y 3.- Presión social, representa la continua insatisfacción a las acciones no legitimas o la inacción del poder para gestionar apropiadamente espacios y respuestas vinculados a la solución de los problemas.

Si bien es cierto una política pública debe conducir a solucionar problemas de carácter global, con principios de inclusión y de representación mayoritaria, también no es menos cierto que muchos de los problemas sociales nacen desde lo más bajo de la 'pirámide espacial”: lo local; justamente desde allí es que deben generarse las soluciones, quien conoce sus necesidades y problemas, seguramente conoce la solución del mismo. En Venezuela es una debilidad, aun cuando existen estructuras oficiales (Consejos Locales de Planificación de Políticas Públicas) para la detección de necesidades locales, estos consejos están minimizados ante la ausencia de protagonismo de la misma y su debilitamiento paulatino por parte de instan- 
cias nacionales, generando debilidades en materia de gobernanza local y una participación ciudadana poco focalizada en sus problemas cotidianos.

Ahora bien, desde el seno de la investigación nos preguntamos, ¿el diseño de una política pública en el contexto local puede representar avances estratégicos en términos de gobernanza y de participación ciudadana? A fin de contrastar, revisamos la opinión de cuatro investigadores destacados en el tema políticas públicas, y sus reflexiones fueron las siguientes:

Es un avance estratégico dado que diseñar una política pública local implica tomar en cuenta al ciudadano, no solo en el ámbito local, que es lo más cercano a la ciudadanía, sino también porque son los espacios naturales de participación ciudadana, y más aún en tiempos de gobernanza, donde se combinan y se reflejan una nueva forma de gobernar donde participan el Estado, el sector privado y los ciudadanos, donde cada quien desde su perspectiva hace su acción, y lo más destacable es que el Estado (El Gobierno local) es un actor facilitador y estimulador de la participación ciudadana y no un interventor (Pérez, 2015, p. 45)

La postura de Pérez (2015) refleja la necesidad de abrir espacios de gobernanza para el diseño de políticas públicas locales, donde el o los factores decisores puedan proponer, dentro del ámbito de su competencia, respuestas a situaciones particulares en pro de un colectivo.

El diseño de una política pública desde el contexto local representa no sólo avance para el Estado y los ciudadanos, sino que permite efectivamente identificar los problemas más sentidos de la localidad, y propiciar un conjunto de alternativas de solución y toma de decisiones desde la experiencia de los ciudadanos inmersos en el tema objeto del diseño de la política, pero sin dejar de lado otras dependencias vinculadas al tema (Rincón, 2015, p. 52)

Rincón (2015) por su parte asume que imprescindible la identificación de problemáticas desde el seno de la sociedad, donde la incorporación de la comunidad donde se encuentra la raíz del problema pueda apuntar hacia la solución sistémica del mismo, generando espacio para la discusión de la política pública.

Esto supone romper con un esquema vertical de relación estadosociedad, para sustituirlo por una relación horizontal. Si esto ocurre se estimula la participación ciudadana lo cual incidirá en la mayor eficacia de la política pública elaborada, con lo cual se estaría cumpliendo con los principios del buen gobierno (Feo La Cruz 2015, p. 63)

Para Feo La Cruz (2015) el buen gobierno parte como icono del ejercicio de la gobernanza y la participación, en las que las decisiones sobre 
políticas públicas deben ser compartidas, lo cual sellaría las bases de una gestión de gobierno transparente y visible ajustada a los requerimientos de la sociedad.

Si consideramos a la política pública como cursos de acción y flujos de información relacionados con la consecución de un objetivo público, y si entendemos el ámbito de lo público como el interés de una diversidad de actores políticos, entre ellos el Estado, el sector privado y la propia comunidad, entonces subrayamos que la participación refiere a la influencia que las personas, ciudadanos, grupos organizados, y comunidad en general, tienen para hacerse presentes en la determinación de la Agenda pública, a través de la exposición de las demandas legítimas (Riquelme, 2015, p. 72)

Riquelme (2015) reflexiona sobre el visibilizar a la ciudadanía y todo aquel actor que represente un punto un valor en las decisiones, donde la inclusión no es un tema de discusión, sino la necesidad de entendimiento entre los influyentes es lo que se debe empezar a valorar, reconociéndose su diversidad y capacidad de aporte.

En resumen podemos acotar que una política pública representa el escuchar las voces de los mudos o de los que no se expresan, permitiendo ser una forma de alertar sobre la importancia que tiene el saber escuchar el clamor de las personas no solo por las necesidades que padecen, sino también de identificar situaciones que comprometen el bienestar del ciudadano al tomar decisiones inconsultas, y peor aún el dejar de auspiciar espacios para el entendimiento y el diálogo. La ciudadanía no organizada desea ser escuchada, muchas veces sus voces se ahogan entre los problemas más simples pero no saben canalizar sus inquietudes; el gobierno local debe proponerse llegar hasta el último espacio de la localidad para que esta sea entendida y atendida por quienes tienen el privilegio de escuchar y dar respuestas. Las decisiones que afectan a la mayoría no deben estar condicionadas por un espíritu diferente al de la cooperación y mancomunidad, solidaridad e inclusión, pluralidad y respeto, así como de un sentido de pertenencia y defensa de la localidad; donde el ejercicio del diálogo permita re-afiliar al propio ciudadano en términos de compromiso ciudadano, asumiendo espacios para el encuentro y el ejercicio constante de prácticas democráticas -consultas- desde el ámbito local.

Es imperante focalizar las dificultades de la comunidad, preguntarse siempre: ¿por qué y cómo nacen los problemas? Debe ser una prioridad enseñar a las comunidades a evitar que los problemas sociales se presenten por falta de información o formación ciudadana, así como de gestionar sus inquietudes y participar en el diseño de la agenda pública; además, procurar concientizar, por la vía de la colaboración y la contribución, el respeto por el ecosistema local que se comparte; como bien dice Giner (1999) “aquello que puede hacer la ciudad no debe de hacerlo el Estado, o aquello que puede hacer una región, no debe hacerlo el Estado” (p. 54). 


\section{Gobernanza}

La temática sobre la gobernanza ha sido muy variada desde que apareció el término, con múltiples definiciones, variadas posturas y diferentes contextos. En esta investigación se procura desde la óptica de la alianza entre sectores vitales de la sociedad, específicamente desde lo local. Vista a partir del ecosistema que forma la localidad para la búsqueda de su crecimiento y sustentabilidad, sin apartarse de su historia, tradiciones, cultura y trascendencia. Se puede entender que la gobernanza "es una estrategia de acercamiento que permite incorporar diversos actores sociales e instituciones gubernamentales o no, en la construcción de modelos autóctonos que permiten visualizar lo local desde la base social, partiendo del consenso/ acuerdo" (Vegas, 2015, p. 322) lo que conlleva, en términos estratégicos, a elaborar una agenda validada por los involucrados en términos de una gestión vinculada a políticas públicas sustentables y de servicios públicos pensados en la ciudadanía, así como también el de propiciar el cuidado o rescate del sentido de identidad de una localidad con ventajas competitivas.

Observando la orientación de lo planteado en el párrafo anterior, se puede deducir que la gobernanza es un proceso de validación de políticas públicas a través de la participación directa de ciudadanos comunes y actores influyentes dentro de un contexto social local común, el cual permite visionar proyectos de sustentabilidad. Ahora bien, ¿es la participación el vehículo idóneo para la construcción de una gobernanza focalizada en políticas públicas locales?

Tras la revisión de los aportes dados por los investigadores y expertos consultados -en el estudio de la gobernanza- nos encontramos con diversas visiones, observemos:

El primer y más importante criterio para mí es el de capacidad política y técnica de gobernanza, es decir, de mecanismos para tomar decisiones con muchos agentes, actores sociales y grupos de interés. Por otro lado, el valor social básico de una democracia me parece es el respeto por la pluralidad y la negociación y deliberación de los objetivos colectivos o públicos (Arellano, 2015, p. 23)

En el ámbito local es muy importante tener en cuenta la participación ciudadana, pues el municipio es la organización del Estado que se encuentra más cerca del ciudadano, lo que permite un mejor conocimiento de la problemática local. Por lo que es necesario agregar a los principios de gerencia, el principio de la subsidiariedad, entendido tanto en la relación del municipio con otras instancias del Estado, en sentido de la distribución vertical del poder, como en la relación Estado-sociedad. En el primer entendido corresponde al municipio, por esa cercanía atender a la solución de los problemas (como primera instancia), pero en atención a sus competencias, por lo que cuando corresponda a otro nivel del Poder Público, el ente local habrá de 
contribuir con las otras instancias. Corresponde al ente local valorar los medios, instrumentos, fórmulas de participación ciudadana, considerando el rol de la familia y de la sociedad en la solución de problemas. No es la ciudadanía la que ha de comprometerse con lo local, sino que las autoridades locales deben tener un compromiso sólido con la localidad (Urdaneta, 2015, p. 25)

Entre principios que privan en la gerencia cuando se trata de formular y ejecutar una política pública es posible mencionar: 1.- La incorporación plena del ciudadano, tanto en la formulación como en la ejecución de la política pública; 2.- La complejidad de las relaciones entre las instituciones y los actores sociales locales; 3.- La creatividad de la gerencia para lograr una transformación institucional capaz de interpretar las demandas de los ciudadanos (Osto, 2015, p. 29)

La gerencia pública local (municipio) debe dar mucha importancia a las comunidades organizadas (clpp) en el proceso de visionar la ciudad debido a que las políticas públicas locales nacen precisamente del diagnóstico sistemático de las comunidades, este proceso de consulta y rendición de cuenta a la comunidad es lo que permite el efectivo resultado (soluciones abordadas), la participación ordenada y formal de los concejos de planificación deben estar integrados de manera formal al órgano de planificación estratégica del gobierno local. Este proceso involucra a la comunidad con los más altos niveles de planificación y de visión de la ciudad. Este proceso construye ciudadanía responsable, involucra a las comunidades, desarrolla sentido de pertenencia, crea cultura de cambio y, sobre todo, crea un proceso de información continua a las comunidades, que mejora sustancialmente la gobernanza de la ciudad. Este proceso compromete a los ciudadanos con lo local (Urriola, 2015, p. 31)

Estas reflexiones aportan a la investigación elementos desde la postura académica, la investigación y gestión. Arellano hace hincapié sobre la necesidad de la pluralidad en el ejercicio de la gobernanza, Urdaneta resalta la importancia del rol del estado en la que debe ser más generoso con la iniciativa privada en términos del principio de subsidiariedad, Osto destaca el rol de la gerencia como catador de demandas por parte de los usuarios, y por último el aporte del ex Alcalde Urriola considera que todo proceso de participación, permite construir ciudadanía, la formación necesaria que debe existir para que se identifiquen las necesidades y se hagan valer los derechos; claro está, contando con el apoyo de la gestión local.

En síntesis se puede manifestar que la Gobernanza es como "mirarse a través de un cristal como espejo", significando la necesidad de mostrar transparencia en todas y cada una de las acciones emprendidas, donde la confianza es tan frágil y muy fácil de romper si los recursos públicos no son bien administrados, y más aún si se “ocultan” espacios para la deliberación sobre el futuro de la localidad en las que todos los actores deben estar comprometidos. Hay coincidencias en que es indispensable tener la a capa- 
cidad de visionar la localidad, así como el de establecer estrategias para desarrollo vinculadas a sus ventajas comparativas y competitivas, pero para ello necesitan del impulso de una gestión pública local comprometida en establecer alianzas que viabilicen una mejor calidad de vida para los ciudadanos.

\section{Gestión pública local}

La gestión pública local en Venezuela, dada la complejidad de sus actividades, es una clara demostración de ciertas y determinadas contradicciones en materia administrativa, cuya tendencia a la desorganización brinda resultados inversos a los esperados por la ciudadanía. Esta situación es lo que se denomina el Bucle Tetralógico, en la que “él mismo está determinado por el orden/desorden/interacciones/organización, sin un inicio o fin preestablecido en la cadena de eventos” (Morín, 2001, p. 41). Claro está que este tipo de organizaciones complejas presta a simple vista una cara que denota unidad/totalidad, la realidad es que en ellas coexisten la insuficiencia, la fractura y la inconsistencia en todos los ámbitos del proceso administrativo en la estructura del gobierno local.

Sin dudas, la gestión pública representa un instrumento de gobierno que permite reducir espacios entre el ciudadano y la autoridad gubernamental, siendo el puente conductor, en ambos sentidos, de demandas sociales y ofertas de políticas públicas en la que las estrategias que se utilizan para abordar y dar respuestas a las peticiones juegan un papel importante al momento del ejercicio de la administración de los recursos. Veamos la interpretación dada por Vegas (2015) sobre lo que representa la gestión pública local para los alcaldes en ejercicio y su propia visión sobre la dimensión expuesta:

La gestión pública para los actores sociales es una estructura diseñada para resolver situaciones complejas que afectan a la ciudadanía, y que por sus propios medios no puede hacer. Su conducta está basada bajo dos vertientes: lo político y lo gerencial; en el primer caso asumir posiciones de liderazgo social, y en el segundo como instrumento de buen gobierno como proyección de lo local (Vegas, 2015, p. 111)

La gestión pública local “es la acción política y gerencial del gobierno municipal, soportada por técnicas modernas de administración, cuyo objetivo es procurar espacios de conciencia social entre la ciudadanía y el gobierno para el diseño conjunto de la localidad, así como orientar sus capacidades y estrategias en pro de una calidad de vida en armonía con el ecosistema local (Vegas, 2015, p. 127)

En resumen, se puede decir que la gestión pública local es un espacio para acortar distancias, con una visión y discurso coherente; por parte de quienes asumen la responsabilidad de dirigir los destinos de la localidad: 
• incorporando liderazgo y un equipo interdisciplinario formado para el ejercicio de la gestión pública,

- mostrando sensibilidad social ante los problemas estructurales y necesidades más básicas de la sociedad,

- aportando soluciones integrales mediante herramientas gerenciales que permitan elevar el nivel de calidad de vida y de sostenibilidad de la localidad (de manera permanente con una agenda comprometida con políticas incluyentes y servicios públicos competitivos vinculados al crecimiento y desarrollo de lo local)

- permitiendo enseñar cómo hacer del buen uso de los recursos públicos, sin dejar de lado la participación, en buena pro, de quienes son los más beneficiados de la acción pública.

\section{De lo empírico a lo sustantivo}

No cabe la menor duda que la gestión pública representa la estructura básica para impulsar y coordinar toda política pública que se procure, y desde su misma visión de proyectista asumir los espacios que brinda la gobernanza establecer las estrategias que permitan un mayor acercamiento del ciudadano en la solución de sus problemas y en el desarrollo propio de su espacio social. La investigación demostró un proceso de saturación y vinculación teórica gracias a la revisión bibliográfica (descriptores semánticos), los aportes recibidos por parte de los entrevistados (alcaldes) y encuestados (investigadores) mediante Delphy, así como la interpretación dada por quien valora estos resultados.

\section{Zumo de lo encontrado. \\ Lo cotidiano como factor emergente de políticas públicas locales}

Las localidades municipales, en Venezuela representan una gran variedad en cuanto a sus condiciones geográficas, demográficas, territoriales, culturales, económicas y de costumbres muy singulares según la región en la que se encuentre; características que permiten asumir que la diversidad no solo es notable en su comportamiento social y ciudadano, sino también desde los resultados de la gestión pública local. Los resultados exitosos de una gestión pública local comparada con otra -cuya similitud en la estructura presupuestaria y financiera son si se quiere idénticas, además de una estructura organizativa impuesta por normas legales a todas por igual- son poco comprensibles. En cada caso es importante hacer notar la presencia del nivel académico y de formación gerencial en la estructura organizativa del gobierno local, así como de los procesos administrativos que se desarrollan en pro de los tiempos de respuesta, así como la calidad de servicios básicos a la que la ciudadanía tiene acceso, y por supuesto el nivel de participación de esta última, cuyo nivel de cultura ciudadana también fue revisado desde la perspectiva del discurso del actor social. 
La gestión pública local tiene un alto compromiso que abarca diversos aspectos, entre ellos está el disponer de información y datos que le permitan adelantarse a los problemas de carácter gerencial, y más aún cuando estos problemas parecieran predecibles dado el comportamiento de la ciudadanía y todo lo que envuelve sus necesidades de satisfacción en materia de servicios públicos. Por ello, la estrategia de la gestión pública debe basarse en procurar el diseño de políticas públicas que nazcan del sentir de la sociedad, del "zapato sucio" del funcionario que recorre las veredas de la comunidad que busca alcanzar los problemas, y no esperar a que estos lo alcancen a él; la búsqueda constante de un acercamiento entre los miembros de la localidad debe ser sobre la base de la verdad y de la justicia, con premisas compartidas en términos de solución integral y comprendida entre los más vulnerables de la sociedad.

Sin ninguna duda, la localidad brinda oportunidades de crecimiento y desarrollo en la medida que las tres dimensiones estudiadas -gestión pública local, gobernanza y participación- actúen en forma coordinada y con suficiente autonomía. Beneficios como el aumento de la calidad de vida privilegiaría al ciudadano dentro del contexto social al que pertenece en virtud de ser más cuidadoso de los espacios y el patrimonio de la localidad; con responsabilidad directa en la toma de decisiones que bien pueden cambiar la dirección de la gestión gubernamental en los términos de políticas locales si estas afectan sus intereses comunitarios y sociales; ser partícipes de la sustentabilidad y rentabilidad local, expresada esta última como la oportunidad de vivir en un espacio que provea salud, seguridad, educación y empleo en condiciones favorables para la familia y el entorno; por ello, es necesario incluir estrategias de gobernanza que permitan acercarse al consenso y validación de la agenda pública local.

El discurso de los actores sociales entrevistados se considera sincero y apegado a sus creencias políticas, cada cual con sus matices en cuanto a la forma de ver las dimensiones estudiadas -gobernanza, participación y gestión pública local- y expuestas a lo largo de la investigación; sin embargo, todos coinciden en la necesidad del diálogo como instrumento de entendimiento para la búsqueda de coincidencias que ayuden al desarrollo de su respectiva localidad; donde desde su óptica la crítica y autocrítica es bienvenida siempre cuando sea con la intención de mejorar, y así debe ser. Una crítica puede ser asumida como una “asesoría” gratis de lo que se hace mal, y si es de alguien no afecto a la gestión tiene mucha más validez, permitiendo su revisión inmediata. Esto amerita mucha madurez en la gestión pública. En próximos trabajos, se destacaran detalles sobre la experiencia de cada alcalde entrevistado y el impacto sobre el diseño de una gestión pública pensada en el ámbito que le rodea, donde la arquitectura gerencial representa un símbolo de calidad de vida y de re-afiliación ciudadana al contexto lo local.

A su vez, lo cotidiano debe ser que la ciudadanía se proponga conquistar más espacios y por ende más responsabilidades en la co-gestión de lo público, esto ayuda a la simplificación de la estructura burocrática en la 
administración pública local, por ende a la construcción de una gestión pública humanizada, sensible a los problemas sociales; protagonista en el diseño de políticas públicas locales autóctonas, vigilantes del desarrollo sustentable de su propio ecosistema, y con la vista puesta en la consolidación de su núcleo familiar dentro del contexto local.

Por último, el principal éxito de la gestión pública local pasa por la sinceridad y transparencia de sus actos. La participación de factores influyentes mediante el ejercicio de la gobernanza permite incorporar dolientes y compromisos con la localidad, en la que se busca contribuir directamente con sus principales fortalezas: habilidad intelectual, laboral y capacidad para gobernar; lo más importante es que el engranaje estratégico funcione proporcionalmente ya que así la localidad se vuelve más competitiva en calidad de vida, con servicios públicos modernos y eficientes. Fin del camino...se abren nuevos espacios... nuevas rutas.

\section{Agradecimientos}

Investigación Doctoral, Doctor en Ciencias Gerenciales y Administrativas. 
Polis, Revista Latinoamericana, $N^{\circ} 48,2017$

\section{Notas}

${ }^{1}$ Es una técnica prospectiva utilizada para obtener información esencialmente cualitativa, pero relativamente precisa, acerca del futuro. 


\section{Bibliografía}

Banco Mundial (2017). Informe sobre el desarrollo mundial. La gobernanza y las leyes. Cuadernillo del Panorama General. Washington DC, Estados Unidos: Banco Mundial

Cátala, J.P. (2001). Gobernabilidad democrática para el desarrollo humano. Marco conceptual y analítico. Revista Instituciones y Desarrollo (10)

Constitución de La República Bolivariana de Venezuela (2000, reformada en febrero de 2009). Los Consejos Locales de Planificación Pública. Publicada en Gaceta Oficial Extraordinaria N 5.453- Caracas, República Bolivariana de Venezuela.

De La Torre, G, y otros (2004). Teoría fundamentada o groundedtheory. Métodos de investigación. Madrid, España: Universidad Autónoma de Madrid

Diesing, P (1971). Patterns of discovery in the social sciences. Chicago, Estados Unidos: Aldine- Atherton, Inc.

Eisner, E. W. (1998). El ojo ilustrado. Indagación cualitativa y mejora de la práctica educativa. Barcelona, España: Editorial Paidós.

Gadamer, H. (1988). Verdad y método. Fundamentos de una hermenéutica filosófica. Salamanca, España: Ediciones Sígueme

Giner, S. (1999). Clase Magistral "La ciudad: ámbito de las virtudes republicanas”. Instalación de la Cátedra Rectoral Alexis de Tocqueville. Valencia, Venezuela.

Glaser, B. (1978). Theoretical sensitivity: Advances in the methodology of grounded theory. Mill Valley C. A, Estados Unidos: Sociology Press.

Morín, E. (2002). Introducción a una política del hombre. Barcelona, España: Editorial Gedisa, S.A.

Ricoeur, P. (1969). El conflicto de las interpretaciones. Ensayos de hermenéutica. Buenos Aires, Argentina: Fondo de Cultura Económica.

Strauss, Anselm, y Corbin (2002). Bases de la investigación cualitativa. Técnicas y procedimientos para desarrollar la teoría fundamentada. Editorial Universidad de Antioquia. Medellín.

Vegas, H. (2009). Políticas Públicas en la Venezuela del Siglo XXI. Dirección de Medios y Publicaciones de la Universidad de Carabobo. Valencia, Venezuela. 
(2015) Gestión pública local, gobernanza y participación. Una visión a partir del discurso de los actores sociales en Venezuela. Visión investigativa sobre participación y gestión pública local (Método Delphi). (Tesis de Doctorado) Universidad de Carabobo. Valencia, Venezuela.

Entrevistas realizadas en 2015 en al marco de la Tesis Doctoral

D. Arellano,

M. Feo La Cruz,

Z. Osto,

A. Urdaneta,

G, Urriola,

W, Pérez,

M. T. Rincón,

B. Riquelme

En H, Vegas (2015) Gestión pública local, gobernanza y participación. Una visión a partir del discurso de los actores sociales en Venezuela. Visión investigativa sobre participación y gestión pública local (Método Delphi). (Tesis de Doctorado) Universidad de Carabobo. Valencia, Venezuela.

Recibido: 10.04 .17

Aceptado: 03.11.17 